\title{
Wave Induced Effects on the Hydrodynamic Coefficients of an Oscillating Heave Plate in Offshore Wind Turbines
}

\author{
Krish Thiagarajan ${ }^{1, *,+}$ (1) and Javier Moreno ${ }^{2, \ddagger}$ \\ 1 Department of Mechanical and Industrial Engineering, University of Massachusetts Amherst, \\ Amherst, MA 01007, USA \\ 2 Marflet Marine, 28014 Madrid, Spain; javiermorenodesalas@gmail.com \\ * Correspondence: kthiagarajan@umass.edu \\ † Legal name: Krish Thiagarajan Sharman; Also Adjunct Faculty, Advanced Structures and Composites \\ Center, University of Maine. \\ $\ddagger$ Iberdrola Scholar, University of Maine.
}

Received: 19 July 2020; Accepted: 14 August 2020; Published: 18 August 2020

\begin{abstract}
The research problem discussed in this paper is of relevance to floating offshore wind turbine design, where heave plates are attached to the columns of a semi-submersible in order to improve vertical plane stability and the power output. Because of the shallow draft of these structures, the heave plates are proximal to the water surface. When subject to vertical plane motions the flow around a plate is altered by the presence of the free surface, resulting in changes in added mass and damping forces. In this paper, we present the experimental results for the added mass and damping coefficients for circular heave plates attached to a column, when oscillating in heave in the presence of oncoming waves. The results tend to indicate that applying the hydrodynamic coefficients obtained from still water experiments for a structure moving in waves may only be an approximation. For different relative phases of the wave and the motion, large variations could occur. We define a modified Keulegan-Carpenter $(K C)$ number that depends on the relative amplitude of motion with respect to the wave. With this definition, the added mass and damping values are seen to be closer to the still water trends. However, at lower $K C$ values, the added mass coefficients could differ by $30 \%$, which can affect natural frequency estimates. Thus, caution needs to be exerted in the selection of hydrodynamic coefficients for heave plates oscillating in proximity to the free surface.
\end{abstract}

Keywords: heave plate; free surface effect; floating offshore wind turbine; hydrodynamic coefficients; added mass; damping coefficient; forced oscillation in waves; Keulegan Carpenter number

\section{Introduction}

Heave plates play an important role in the hydrodynamic behavior of floating offshore wind turbine (FOWT) structures. The concept of heave plates arose from their application in offshore spar production platforms, where their characteristics of increasing heave added mass and damping are exploited in order to maintain heave motion within acceptable limits. In the case of a FOWT, heave plates provide increased added mass in the vertical plane that shifts the platform resonance period away from the wave and wind-induced excitation periods and increase the total damping of the platform by enhancing the vortex shedding process [1]. Some prototype designs, e.g., Windfloat [2] (Figure 1) or a spar [3] use heave plates to stabilize the platform in pitch, thus improving the power output of the wind turbine.

Experimental research is the main means to study the hydrodynamic characteristics of heave plates, although some computational fluid dynamics solutions have also been reported (see e.g., $[4,5]$ ). 
Scaled model tests can provide a good understanding on the behavior of floating platforms at various stages of design and development. This reduces risks and helps to optimize the design of the prototype platform. Hydrodynamic data on heave plates are usually reported using two non-dimensional characteristic parameters (Keulegan-Carpenter number $K C$ and frequency parameter $\beta$ ) that represent the amplitude and frequency of oscillation of any heave plate configuration [6-10]. These dimensionless parameters of relevance are defined as [11]:

$$
\begin{gathered}
K C=\frac{2 \cdot \pi \cdot Z_{0}}{D_{d}} \\
\beta=\frac{D_{d}^{2} \cdot f}{v}
\end{gathered}
$$

Here, $D_{d}$ is the disk diameter, $Z_{0}$ and $f$ are, respectively. the heave amplitude and frequency of oscillation, and $v$ is the kinematic viscosity of the fluid.

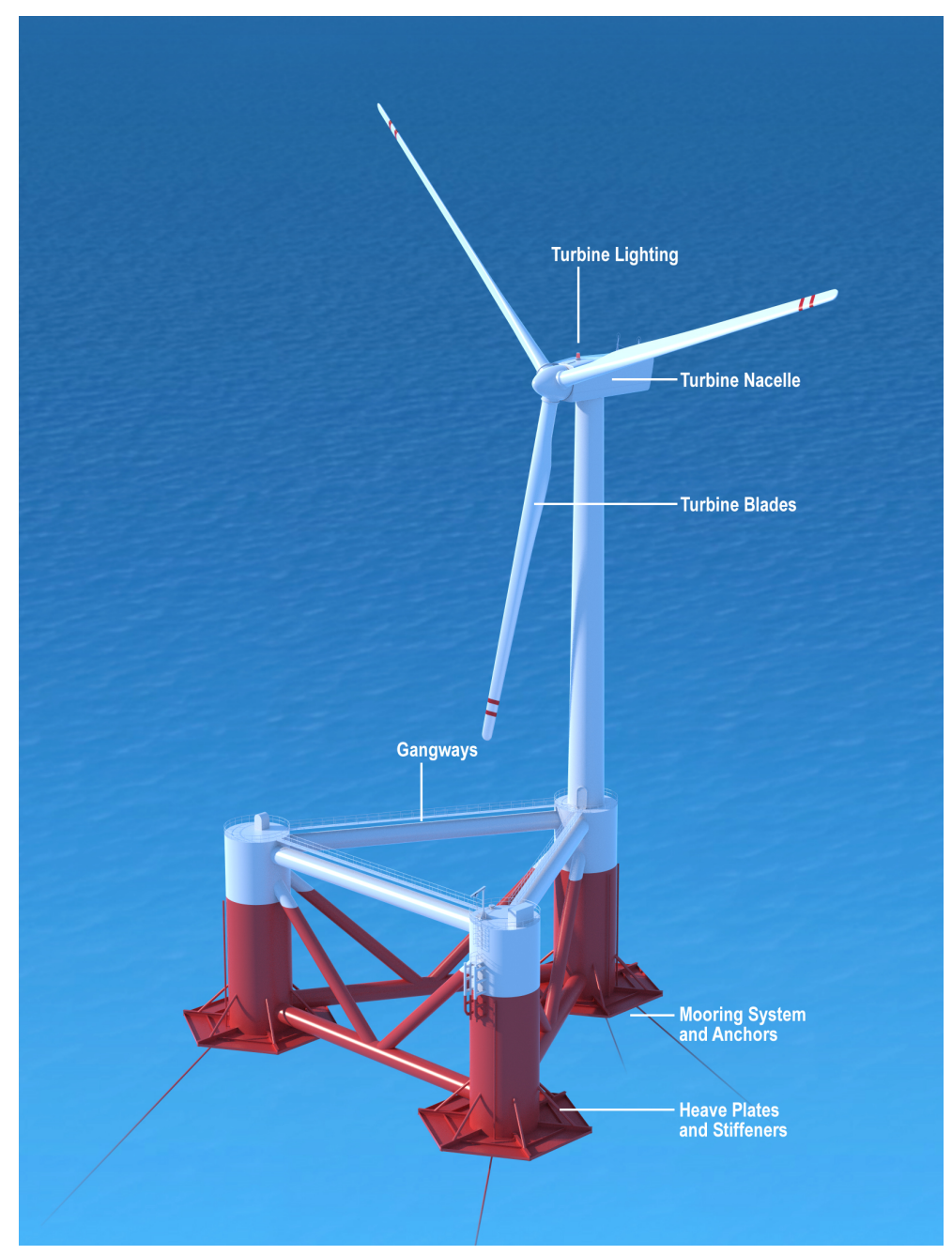

Figure 1. A floating offshore wind turbine showing heave plates attached to the structural columns [2]. Attributed to Untrakdrover/CC BY-SA (https://creativecommons.org/licenses/by-sa/3.0).

Studies on the hydrodynamic coefficients of structures with heave plates have focused on obtaining values of these coefficients at parametric ranges of importance, as well as on behavioral trends with respect to $K C$ and $\beta$. Thiagarajan and Troesch in [7] conducted model tests on circular columns of a TLP platform. It was found that, while the added mass coefficient was invariant at low 
$K C$ numbers at a fixed oscillation frequency, the heave damping coefficient was found to be linear when plotted versus $K C$, and was made up of two components:

- Friction drag: damping obtained by integrating the shear stress over the wet surface of the body. This damping is dependent on viscosity, hence on Reynolds number or $\beta$. It is negligible except at very small $K C$ numbers.

- Form drag: term due to flow separation and vortex shedding at the edges. It is obtained by integrating the normal stresses over the wet surface of the body. It is highly influenced by the geometry of the body. They found this term to be linear with the amplitude of oscillation.

The same linear tendency of damping when plotted against $K C$ was also found in [12]. In this study, experimental tests at low $K C$ were performed on a circular heave plate of $0.609 \mathrm{~m}$ diameter attached to a column of $0.457 \mathrm{~m}$ diameter. The experiments were performed at 1:75 scale by [8] on a spar platform with two circular heave plates of $0.68 \mathrm{~m}$ and $0.60 \mathrm{~m}$ diameter. They also found that the heave damping was primarily form drag for low $\beta$ numbers. The effect of $\beta$ was found to be small for small $K C$.

On the matter of vortex shedding flows, Tao and Thiagarajan [5] studied the viscous flow around an oscillating cylinder with a heave plate by direct numerical simulations. Flows at $K C$ numbers ranging from $7.5 \times 10^{-4}$ to 0.75 and at $\beta=1.585 \times 10^{5}$ were studied. Three different shedding modes were found, i.e. independent, interactive, and uni-directional vortex shedding. The occurrence of these modes was shown to be dependent on $K C$ and the aspect ratio represented by the ratio between the disk thickness $t_{d}$ and the disk diameter $D_{d}$. The vortex shedding was found to be uni-directional for thinner disks at low $K C$. A quantitative method of identifying the vortex shedding flow regimes based on $K C$ and aspect ratio was presented in [6]). A distinct increase in damping, depending on the vortex shedding regime, was also observed.

The added mass effect was examined in detail in a number of publications. The added mass coefficient was found to have a weakly linear trend as the range of $K C$ was increased. The added mass of a circular plate attached to and separated from a column was studied by [13]. The added mass coefficient was found to double when the plate was separated from the disk, but still largely invariant with KC. A similar observation was also found by [14], whose experiments covered a range of separation distances between the column and the plate. The authors also provided theoretical formulations for the added mass coefficient as a function of the separation distance.

The above observations largely apply to solid heave plates submerged in water and oscillating in isolation. On the other hand, proximity to a boundary, thickness to width ratio, the shape of the edge, and porosity of the heave plates can alter the behavior of the hydrodynamic coefficients. In [9], experiments with $0.4 \mathrm{~m}$ rectangular heave plates were performed with different submergence from the free surface ranging from 0.4 to $1.2 \mathrm{~m}$. The results showed that the added mass increased linearly with $K C$ but showed indifferent trend over the range of submergence tested (whose minimum value was one diameter). The drag coefficient-which is related to damping coefficient over $K C$-was found to vary inversely with $K C$, but showed similar invariant behavior with submergence. In all these cases, the effect of changing $\beta$ was negligible over the ranges tested. Furthermore, the effect of ambient currents on the hydrodynamic coefficients of a plate attached to a column has also been studied (see e.g., [12]).

When a heave plate is brought closer to a surface either by increasing the $K C$ value or by reducing the draft of the attached column, then some changes become apparent. Numerical studies by [10] have shown that vortices shed by heave plates when executing large amplitude oscillations can disturb an otherwise quiescent free surface. They also showed that both coefficients exhibited dramatic variations with increasing $K C$, which depended on the distance from the free surface. A similar behavior was also observed when a plate was moved close to a solid surface like a seabed. Energy dissipation arguments were used to explain damping variations that were observed when amplitude of oscillation was changed or when the free surface was proximal. 
This paper arose out of an interest to know how waves on the free surface will alter the hydrodynamic coefficients of heave plates. To study this problem, we consider the forced heave motion of a column with a heave plate in the presence of waves. The problem is of relevance to floating offshore wind turbine design, where heave plates are attached to the columns of a semi-submersible. Because of the shallow draft of these structures, the heave plates are proximal to the water surface and, hence, wave induced water motions could affect the hydrodynamic behavior. In the next section, we present some theoretical background, followed by details of an experimental study that was conducted for this research.

\section{Theoretical Model}

We initially consider a structure that is forced to harmonically oscillate in the vertical direction, $Z$ (heave) in still water, Figure 2. Using Newton's second law, the force $F_{33}$ that is required to move the structure is shown as Equation (3):

$$
F_{33}(t)=\left(M+A_{33}\right) \ddot{Z}+B_{33} \dot{Z}+K_{33} Z
$$

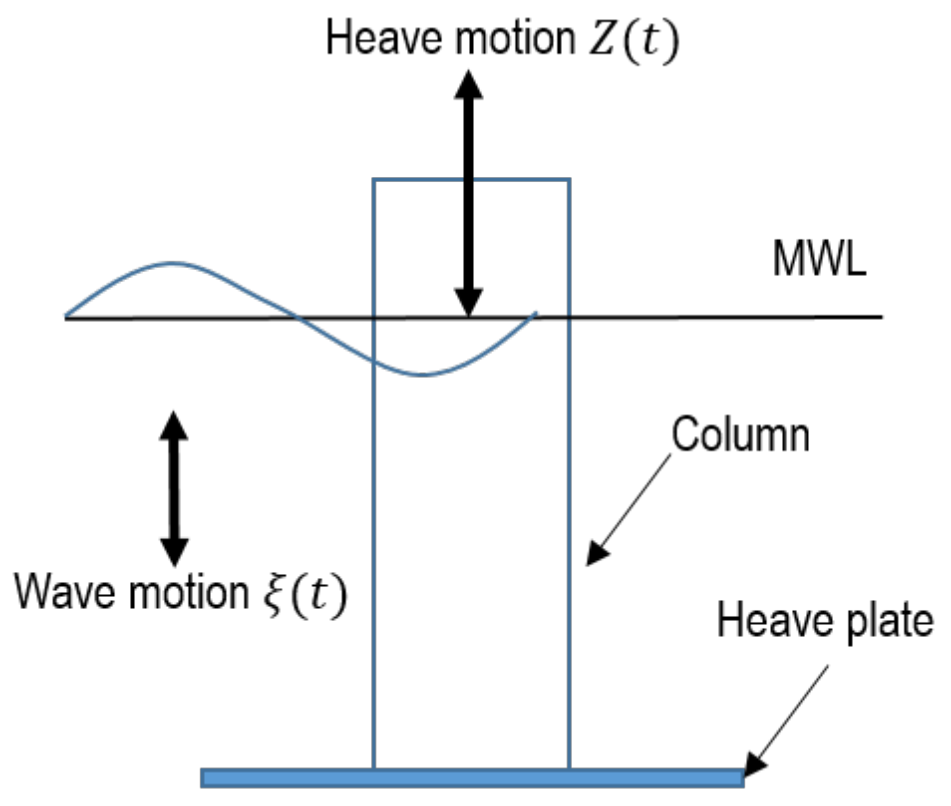

Figure 2. Schematic of a column with heave plate showing motions of relevance.

Subscript 3 denotes the heave direction, $M$ and $A_{33}$ are the mass and heave added mass of the body, respectively, and $B_{33}$ is the linearized heave damping coefficient. $K_{33}=\rho g A_{w}$ is the heave hydrostatic restoring coefficient that depends on the water plane area $A_{w}$. The non-dimensional hydrodynamic coefficients are defined as:

$$
A_{33}^{\prime}=\frac{A_{33}}{A_{33_{t h}}} ; B_{33}^{\prime}=\frac{B_{33}}{2 \pi f \cdot A_{33_{\text {th }}}}
$$

where $A_{33_{\text {th }}}=\frac{1}{12} \rho\left(2 D_{d}^{3}+3 \pi D_{d}^{2} z-\pi^{3} z^{3}-3 \pi D_{c}^{2} z\right)$ is the theoretical added mass for a column with a disk attached at the bottom [15]. $D_{c}$ and $D_{d}$ are the column and disk diameters, respectively, and $z=\frac{1}{\pi} \sqrt{D_{d}^{2}-D_{c}^{2}}$.

The damping forces typically have a linear and a quadratic component [12]. By using a linearized damping coefficient, the nonlinear effects are translated into a varying dependence on the coefficients $K C$ and $\beta$. A typical forced oscillation experiment in still water can be conducted in order to evaluate this dependence. A least squares method [11] can be used to find the optimum hydrodynamic 
coefficients, $A_{33}$ and $B_{33}$, which minimizes the error between the measured force during experiments $\left(F_{\text {exp }}\right)$ and the heave force $\left(F_{33}\right)$ over $n$ samples (Equation (3)):

$$
\begin{gathered}
E^{2}=\sum_{i=1}^{n}\left(F_{\text {exp }_{i}}-F_{33_{i}}\right)^{2} \\
\frac{d E^{2}}{d A_{33}}=0 \\
\frac{d E^{2}}{d B_{33}}=0
\end{gathered}
$$

The only unknowns in Equation (5) are $A_{33}$ and $B_{33}$. The displacement and the force are measured during the experiments. The velocity and acceleration can be obtained by numerically differentiating the displacement. By solving Equations (6) and (7), we obtain a system of two equations and two unknowns $\left(A_{33}\right.$ and $\left.B_{33}\right)$, which can be solved numerically.

A windowing method was used during the least squares process. In this method, the added mass and damping are evaluated in different cycles. First, the acceleration signal is divided into different windows with each one containing an acceleration peak. For each window, the added mass and damping are evaluated using the least squares method. The obtained damping coefficient is rejected at this stage, since it is poorly evaluated at acceleration peaks. This process is repeated over 32 cycles yielding the mean and the standard deviation of each coefficient through statistical analysis. The same procedure is done with the damping coefficient while using the velocity signal. This time the added mass is rejected, keeping the damping coefficient. This method was also used in [16].

The relative kinematics between the platform and the water particles need not be considered when the platform is oscillating in still water. When the platform is oscillating in waves, the presence of the wave field alters the water particle kinematics near the edge of a heave plate. The resulting effect is complicated by the fact that the wave field itself is altered by the presence of the oscillating object. Furthermore, it is apparent that the motion of the object is caused by the forcing due to waves. In a linear sense, one can distinguish between radiation and incident/diffraction problems. In order to obtain suitable hydrodynamic coefficients for solving the radiation problem, one can use a linearized version of the relative velocity model described in [17],

$$
F_{33}(t)=M \ddot{Z}+A_{33}(\ddot{Z}-\ddot{\xi})+B_{33}(\dot{Z}-\dot{\xi})+K_{33} Z+\frac{\pi D_{d}^{2}}{4} P_{b}-\frac{\pi}{4}\left(D_{d}^{2}-D_{c}^{2}\right) P_{t}
$$

where $P_{t}$ and $P_{b}$, respectively, represent the wave dynamic pressure acting on the top and bottom of the heave plate, and $\dot{\xi}$ and $\ddot{\xi}$ are the water particle vertical velocity and acceleration respectively at the mean position of the plate. It can be verified that accounting for dynamic pressures on both surfaces of a disk is equivalent to using a relative motion in the restoring force term, i.e., $K_{33}(Z-\xi)$.

If the wave kinematics at the mean position of the plate can be measured or estimated satisfactorily using a wave theory, then one can replace the Equation (3) with Equation (8) in the least squares analysis described above. One can then imagine two different approaches to evaluate the added mass and damping coefficients in the presence of waves:

- Absolute model: this model is the same as the one used to evaluate the coefficients in still water. In this model the waves are not taken into account explicitly. Therefore, a variation is expected when comparing the obtained coefficients in waves with the ones obtained in still water. This model is akin to the default approach, and consistent with the linear superposition of radiation and incident wave problems.

- Relative model: this model takes into account the presence of waves interacting with the heave plate. It includes the Froude-Krylov forces as well as the relative kinematics between the heave plate and the water particles. Here, the coefficients are explicitly made dependent on 
wave kinematics. This approach may be considered to be more appropriate for evaluating suitable coefficients.

In the vicinity of a heave plate, the phase difference between the plate motion and the wave-induced water particle motion becomes a key parameter in the relative model. Let us, for example, consider the relative motion as:

$$
Z_{r e l}(t)=Z(t)-\xi(t)
$$

where

$$
\begin{aligned}
& \xi(t)=\xi_{0} \cos \left(w t+\alpha_{w}\right) \\
& Z(t)=Z_{0} \cos \left(w t+\alpha_{p}\right)
\end{aligned}
$$

By simple rearrangement, we can obtain

$$
Z_{r e l}(t)=A_{r e l} \cdot \cos (w t+\alpha)
$$

where,

$$
A_{\text {rel }}=\sqrt{Z_{0}^{2}+\xi_{0}^{2}-2 Z_{0} \xi_{0} \cos (\alpha)}
$$

and $\alpha=\alpha_{w}-\alpha_{h p}$. Thus, one could define a "relative" KC number

$$
K C_{w}=\frac{2 \cdot \pi \cdot A_{r e l}}{D_{d}}
$$

This is similar to the relative velocity based $K C$ number mentioned in [17].

\section{Experimental Setup}

A circular heave plate of diameter $0.25 \mathrm{~m}$ and thickness $4.3 \mathrm{~mm}$ attached to a column of diameter $0.088 \mathrm{~m}$ and draft $0.19 \mathrm{~m}$ is considered. This model is a 1:80 scaled version of a demonstration prototype off the coast of Spain reported in $[18,19]$. The experiments were performed at the Marine Ocean and Offshore Research (MOOR) wave tank facility at the University of Maine, which is $8 \mathrm{~m}$ long and $1 \mathrm{~m}$ wide. The water depth for the experiments was kept at $0.7 \mathrm{~m}$. A wedge-shaped plunger type wave maker was installed at one end, and a passive energy absorbing beach at the other end. The wave maker is capable of producing regular waves from $0.5-2 \mathrm{~s}$ periods and amplitudes ranging from $0.002-0.132 \mathrm{~m}$. The beach design was optimized to produce reflection of $5-10 \%$ over most of the range of testing.

Forced harmonic oscillation of the models in the vertical direction was achieved while using a Parker ETH032 linear actuator driven by a 750 W Parker servo motor. Two Omega force sensors were attached by two slender rods to measure the vertical forces (Figure 3). The heave displacement was measured by a string potentiometer. Output signals were amplified, sampled, and acquired at $1 \mathrm{kHz}$. Using the least squares approach, the optimum hydrodynamic coefficients, $A_{33}$ and $B_{33}$, which minimize the error between the measured force during experiments $\left(F_{\text {exp }}\right)$ and heave force $\left(F_{33}\right)$ are found. A 32-cycle windowing method described in the previous section was used in the added mass and damping evaluation. 


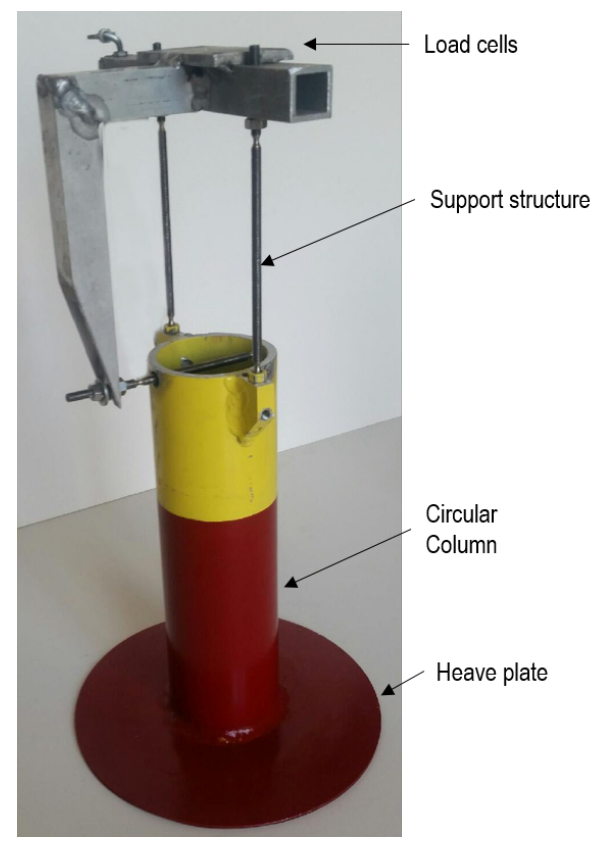

Figure 3. Column with a circular heave plate attached to a frame.

The first set of experiments was conducted in still water. The model was forced to oscillate over a range of $K C$ values from $0.05-1.2$ at a frequency of $1 \mathrm{~Hz}(\beta=62251)$. At larger oscillation amplitudes, disturbance caused by the motion on the free surface radiated outwards. Two triangular wave deflectors were located on each of the tank walls at the heave plate location to reduce reflection from the side walls arising from the disturbance (Figure 4). This simple device performed satisfactorily, as evidenced by Fourier analysis of the force time histories. This showed that several spurious peaks were present in the time histories recorded without the deflectors.

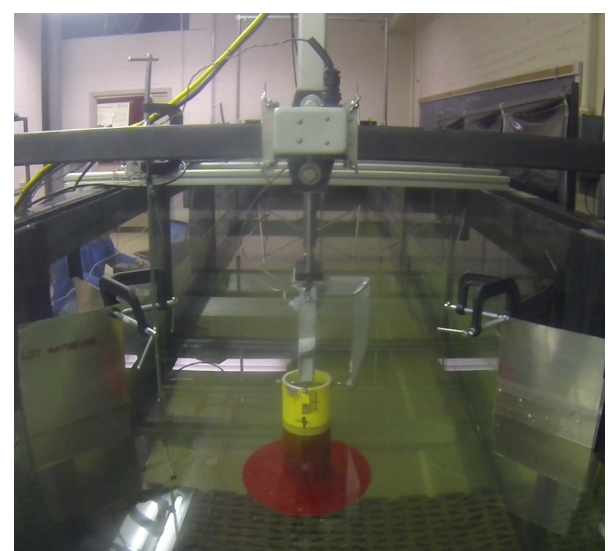

Figure 4. Photo showing the Marine Ocean and Offshore Research (MOOR) wave tank with the model and actuator in the foreground. Wave deflectors on either side for still water runs.

The second set of experiments were conducted in waves. The model was forced to oscillate at a frequency of $1 \mathrm{~Hz}$ and two $K C$ values of 0.5 and 0.84 . The wave frequency was set at $1 \mathrm{~Hz}$ to match the heave plate oscillation frequency. The wave steepness varied from $H / L=0.018-0.02$. Table 1 presents the experimental test matrix. The phase difference between the wave and the platform motion was introduced manually by visual observation of the first three waves measured by a probe located adjacent to the model. The heave plate motion was triggered when the third wave crest reached a desired distance from a zero phase mark. This approach resulted in several runs at different phases ranging from $\alpha=0^{\circ}$ to $\alpha=360^{\circ}$. Each experiment was repeated for different phases in between this range in order to study the effects of the phase between the platform and the wave. In order to 
calculate the phase between the wave and the heave plate signal, a frequency analysis was performed using the platform displacement and the wave elevation signals, such as shown in Figure 5.

Table 1. Wave-heave plate interaction test matrix. $\mathrm{H} / \mathrm{L}$ is the wave steepness.

\begin{tabular}{ccc}
\hline $\mathbf{f} \mathbf{( H z )}$ & $\mathbf{H} / \mathbf{L}$ & $\boldsymbol{K C}$ \\
\hline 1 & 0.020 & 0.84 \\
1 & 0.018 & 0.84 \\
1 & 0.018 & 0.5 \\
\hline
\end{tabular}

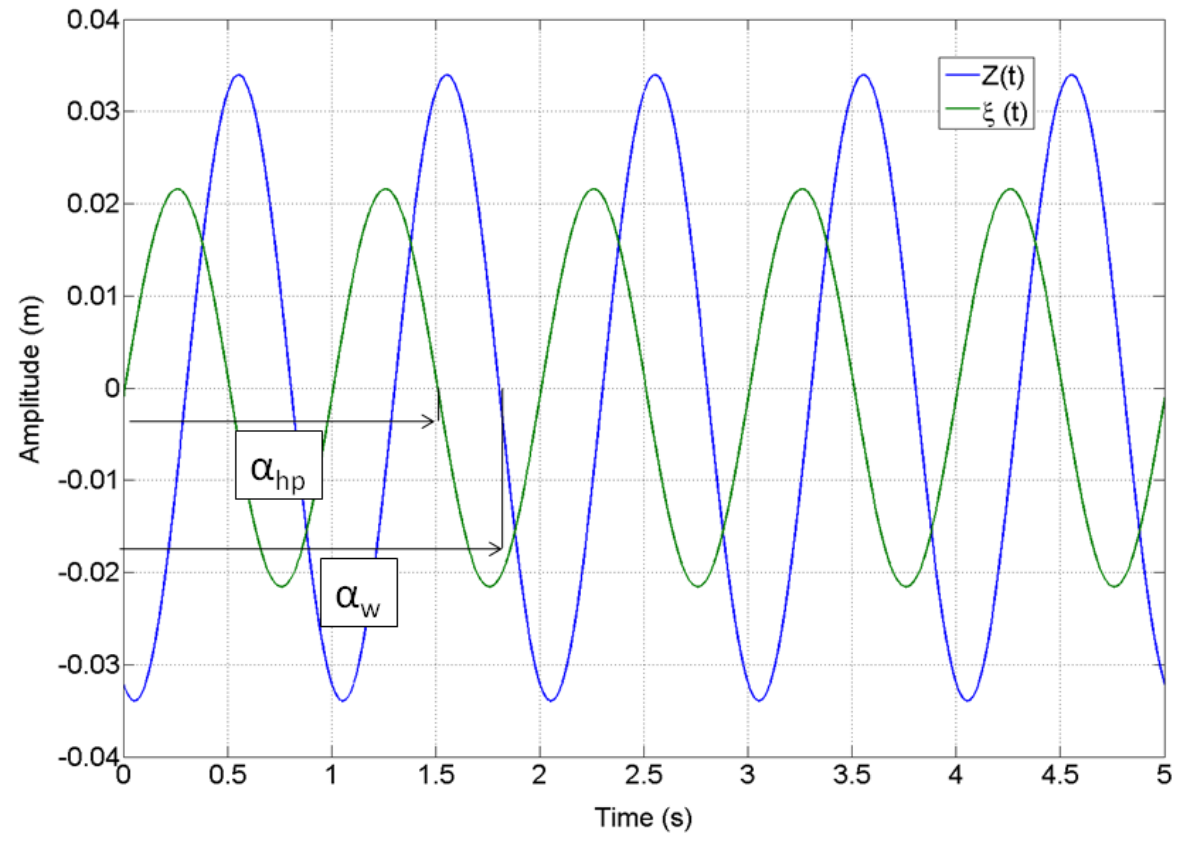

Figure 5. Wave and heave plate phase.

The quality of the generated wave was tested with a repeatability test. The platform was replaced with a third wave probe to measure the wave field at the exact location of the platform. Each wave case was repeated three times. For each case, a statistical analysis was performed that yielded the mean and standard deviation of the wave amplitude and frequency.

\section{Results}

The added mass and damping coefficients for the wave experiments were obtained by two different approaches. In the "Absolute model" approach, Equation (3) is used in the least squares evaluation. This model is identical to the still water case, and all wave-induced variations were visible in the trends of the coefficients with the phase angle. In the "Relative model" approach, Equation (8) which incorporates relative kinematics is used. Sample added mass and damping results using the two equation models are shown in Figures 6 and 7 for different phase angles at $H / L=0.02$ and $K C=0.84$. At this $K C$ value, still water added mass and damping values are 1.42 and 0.85 , respectively. 


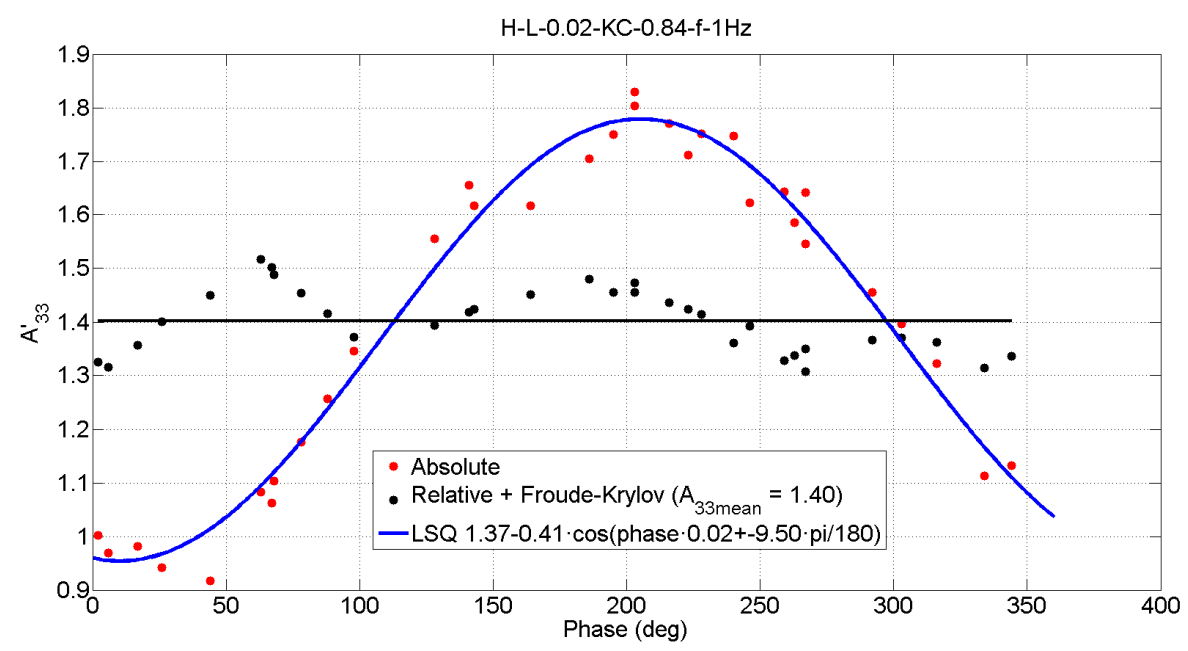

Figure 6. Added mass coefficient vs. phase angle for $H / L=0.02, K C=0.84$.

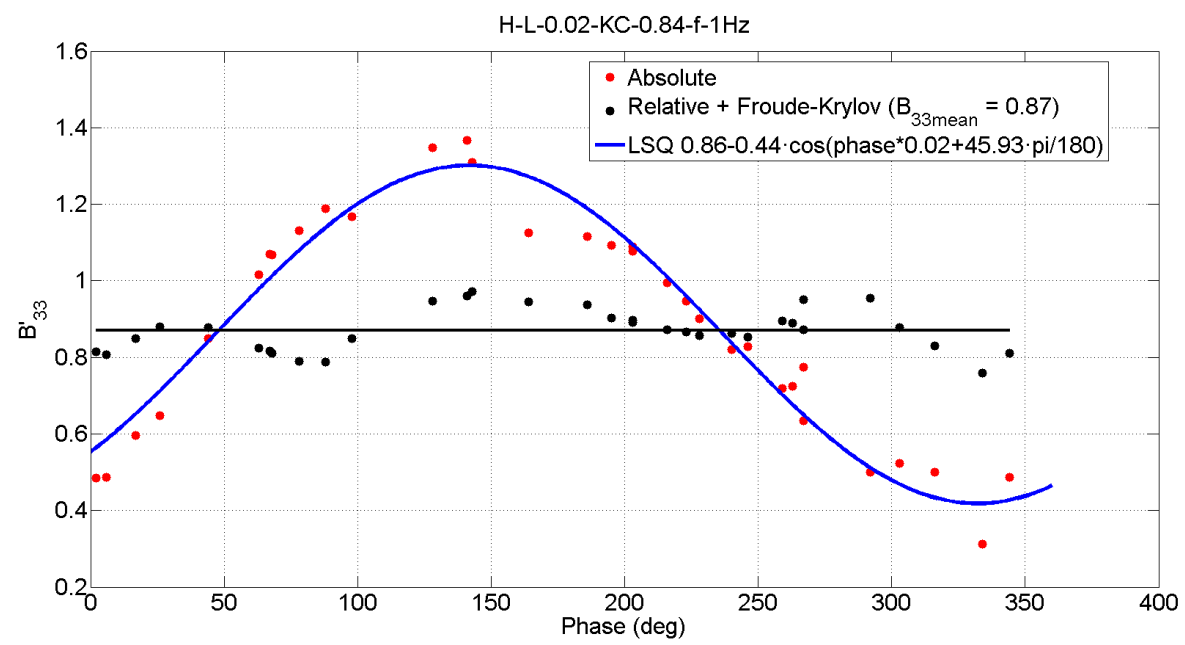

Figure 7. Damping coefficient vs. phase angle for $H / L=0.02, K C=0.84$.

When using the absolute model approach, a clear sinusoidal trend is observed with respect to the phase angle. Interestingly, the mean value of this sinusoidal variation matches with the corresponding still water added mass and damping values to within 3\%. When the relative flow approach is used, the trend of both coefficients with the phase is much flatter, tending towards a constant value that matches the still water value to within $4 \%$.

The relative phase between the plate and the wave gives rise to a relative change in the $K C$, although the amplitude of oscillation is kept constant. Figures 8 and 9 present the added mass and damping coefficients obtained using the relative model against $K C_{w}$. Additionally shown are the results obtained in still water for the added mass and damping coefficients vs. KC. The results are presented for the cases $K C=0.84$ and $K C=0.5$ for a frequency of oscillation of $1 \mathrm{~Hz}$ and for $H / L=0.018$ and $H / L=0.02$. The observed linear trend in the coefficients is remarkable. It can be seen that the added mass and damping coefficients increase as the relative displacement between the plate and the wave particles increases. The added mass coefficients in waves show a steeper linear trend when compared with the still water coefficients. For small $K C$, the added mass coefficients in still water are higher. As $K C$ increases, the coefficients in waves become slightly higher than the ones in still water. The damping coefficients in still water and in waves are very similar in slope, with the zero offset showing a difference. 


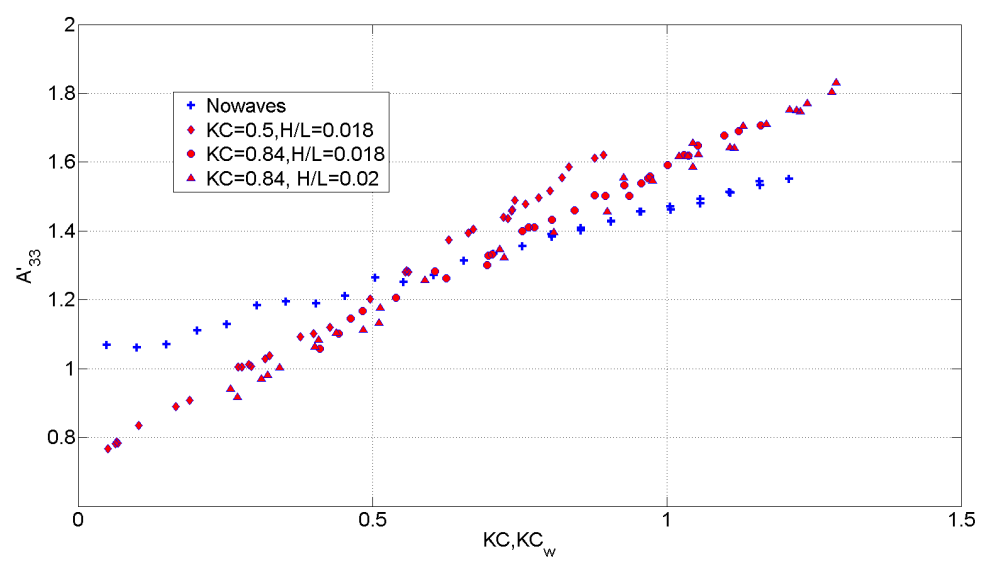

Figure 8. Comparison of the added mass coefficients in waves vs. $K C_{w}$ with those in still water vs. $K C$.

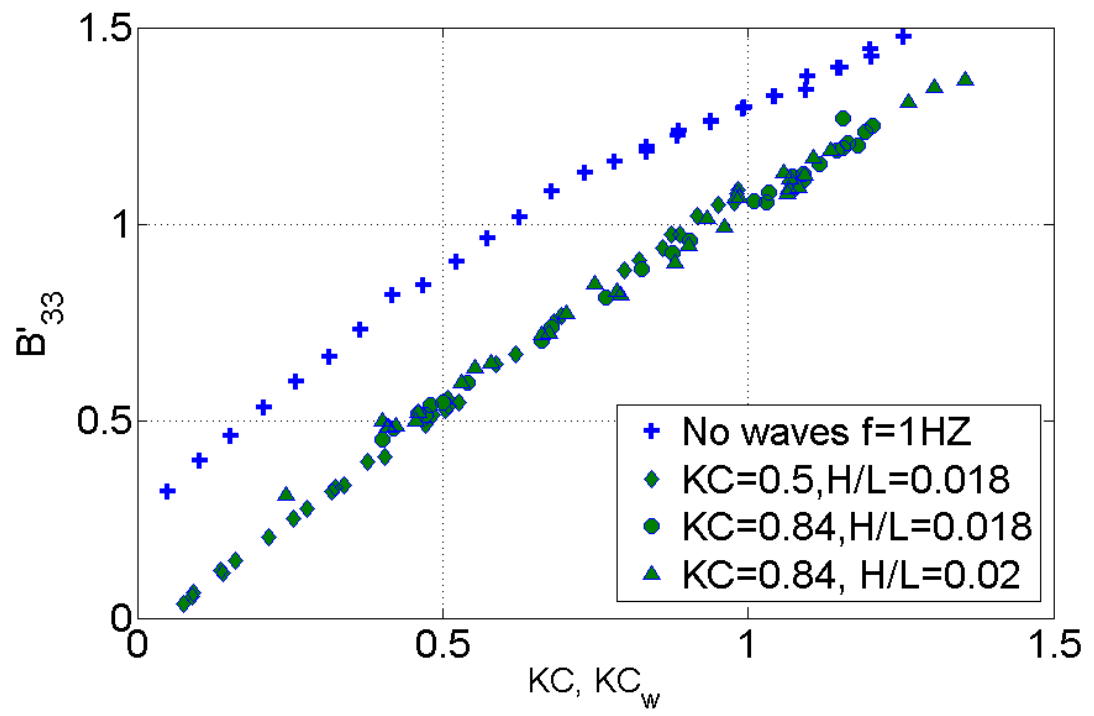

Figure 9. Comparison of the damping coefficients in waves vs. $K C_{w}$ with those in still water vs. $K C$.

\section{Discussion and Conclusions}

This paper has focused on the effect of ambient wave motion on the hydrodynamic forces acting on an oscillating heave plate. When compared to the plate oscillating in still water, large differences in the values of the added mass and damping coefficients are observed. These differences are quite pronounced when the relative motion between the water and the plate are not taken into account. The results from Figures 6 and 7 tend to indicate that applying the added mass and damping coefficients obtained from still water experiments for simulating the motion of a structure in waves may lead to inconsistent results. However, due to scarcity of data on oscillating plates in waves, one method of getting reliable added mass and damping values would be by using the newly defined $K C_{w}$, which depends on the relative amplitude of motion with respect to the wave. As seen in Figures 8 and 9 , the trends between the results in waves are somewhat closer to those that were obtained in still water.

Because damping values are more critical in estimating the maximum motions around resonance, a relative phase angle of $\pi / 2$ may be used for $K C_{w}$. This could be used iteratively along with motion magnitude to find the optimum damping coefficient. On the other hand, it is seen in Figure 6 that, at around a phase angle of $90^{\circ}$, the added mass coefficients in waves and in still water are similar in magnitude. However, added mass coefficients are of relevance in all motion ranges. From Figure 8 , it is seen that, at lower $K C$ values, the added mass coefficients could differ by $30 \%$, which can affect inertial load calculations. Thus, caution needs to be exerted in selection of hydrodynamic 
coefficients for heave plates oscillating in proximity to the free surface. More data would support better estimates of hydrodynamic coefficients for use in simulation of offshore wind turbine platform motions. Future work by the researchers would include a broader range of wave parameters and oscillation ranges. It is also envisaged that currents could be added to the environment in order to understand the combined effect of waves and currents.

Author Contributions: Conceptualization, K.T.; methodology, K.T. and J.M.; experimentation and validation, J.M.; analysis, J.M. and K.T.; resources, K.T.; writing-original draft preparation, K.T.; writing-review and editing, K.T. and J.M.; project administration, K.T.; All authors have read and agreed to the published version of the manuscript.

Funding: This research received no external funding.

Acknowledgments: The paper is a development of an earlier version presented at the 36th International Workshop on Water Waves and Floating Bodies (IWWWFB36) held in Plymouth, MI. The authors acknowledge the support of Stephen Abbadessa, Matthew Cameron and Raul Urbina, Department of Mechanical Engineering, University of Maine in support of the MOOR laboratory. The second author acknowledges the financial support of Iberdrola Foundation for his fellowship during the study.

Conflicts of Interest: The authors declare no conflict of interest.

\section{Abbreviations}

The following abbreviations are used in this manuscript:

$\alpha \quad$ Phase difference between the wave and the plate motion

$\beta \quad$ Frequency parameter

$v \quad$ Kinematic viscosity of water

$\xi \quad$ Water particle vertical displacement

$\xi_{0} \quad$ Water particle vertical displacement amplitude

$\rho \quad$ Mass density of water

$A_{33} \quad$ Added mass in heave

$A_{33_{\text {th }}} \quad$ Theoretical added mass in heave

$A_{33}^{\prime} \quad$ Added mass coefficient in heave

$A_{r}$ el Relative motion between wave and plate

$A_{w} \quad$ Water plane area of the column

$B_{33}^{\prime} \quad$ Non-dimensional damping coefficient in heave

$B_{33} \quad$ Damping coefficient in heave

$D_{c} \quad$ Column diameter

$D_{d} \quad$ Heave plate diameter

$f \quad$ frequency of oscillation

$F_{33} \quad$ Heave exciting force

$F_{\text {exp }} \quad$ Heave force measured in an experiment

$H \quad$ Wave height

$K_{33} \quad$ Restoring force coefficient in heave

KC Keulegan-Carpenter Number

$K C_{w} \quad$ Relative Keulegan-Carpenter Number

$L \quad$ Wave length

M Mass of the oscillating structure

$P \quad$ wave-induced dynamic pressure

$Z \quad$ Heave displacement

$Z_{0} \quad$ Heave motion amplitude

$Z_{\text {rel }} \quad$ Relative displacement between wave and plate motion in the vertical direction 


\section{References}

1. Subbulakshmi, A.; Sundaravadivelu, R. Heave damping of spar platform for offshore wind turbine with heave plate. Ocean Eng. 2016, 121, 24-36. [CrossRef]

2. Roddier, D.; Cermelli, C.; Aubalt, A.; Weinstein, A. WindFloat: A floating foundation for offshore wind turbines-Part I: Design basis and qualification. J. Renew. Sustain. Energy 2010, 2, 33104. [CrossRef]

3. Liu, L.; Guo, Y.; Jin, W.; Yuan, R. Motion performances of a 5 MW VAWT supported by spar floating foundation with heave plates. In Proceedings of the ASME 2017 36th International Conference on Offshore Mechanics and Arctic Engineering, Trondheim, Norway, 25-30 June 2017; Paper 62625.

4. Zhang, S.; Ishihara, T. Numerical study of hydrodynamic coefficients of multiple heave plates by large eddy simulations with volume of fluid method. Ocean Eng. 2018, 163, 583-598. [CrossRef]

5. Tao, L.; Thiagarajan, K. Low KC flow regimes of oscillating sharp edges I. Vortex shedding observation. Appl. Ocean Res. 2003, 25, 21-35. [CrossRef]

6. Tao, L.; Thiagarajan, K. Low KC flow regimes of oscillating sharp edges. II. Hydrodynamic forces. Appl. Ocean Res. 2003, 25, 53-62. [CrossRef]

7. Thiagarajan, K.P.; Troesch, A.W. Hydrodynamic heave damping estimation and scaling for tension leg platforms. J. Offshore Mech. Arctic Eng. 1994, 116, 70-76. [CrossRef]

8. Thiagarajan, K.P.; Datta, I.; Ran, A.Z.; Tao, L.; Halkyard, J.E. Influence of heave plate geometry on the heave response of classic spars. In Proceedings of the ASME 21st International Conference on Offshore Mechanics and Arctic Engineering, Oslo, Norway, 23-28 June 2002; pp. 621-627.

9. Li, J.; Liu, S.; Zhao, M.; Teng, B. Experimental investigation of the hydrodynamic characteristics of heave plates using forced oscillation. Ocean Eng. 2013, 66, 82-91. [CrossRef]

10. Garrido-Mendoza, C.A.; Thiagarajan, K.P.; Souto-Iglesias, A.; Bouscasse, B.; Colagrossi, A. Numerical investigation of the flow features around heave plates oscillating close to a free surface or seabed. In Proceedings of the ASME 2014 33rd International Conference on Offshore Mechanics and Arctic Engineering, San Francisco, CA, USA, 8-13 June 2014.

11. Sarpkaya, T.; Isaacson, M.d.S.Q. Mechanics of Wave Forces on Offshore Structures; Van Nostrand Reinhold Company: New York, NY, USA, 1981.

12. Thiagarajan, K.P.; Troesch, A.W. Effects of appendages and small currents on the hydrodynamic heave damping of TLP columns. J. Offshore Mech. Arctic Eng. 1998, 120, 37-42. [CrossRef]

13. Lake, M.; Troesch, A.W.; Perlin, M.; Thiagarajan, K.P. Scaling effects in hydrodynamic coefficient estimation of TLP and spar structures. J. Offshore Mech Arctic Eng. 2000, 122, 118-124. [CrossRef]

14. Zhu, L.; Lim, H.-C. Hydrodynamic characteristics of a separated heave plate mounted at a vertical circular cylinder. Ocean Eng. 2017, 131, 213-223. [CrossRef]

15. Tao, L.; Molin, B.; Scolan, Y.-M.; Thiagarajan, K. Spacing effects on hydrodynamics of heave plates on offshore structures. J. Fluids Struct. 2007, 23, 1119-1136. [CrossRef]

16. Beatty, S.; Buckham, B. Experimental comparison of self-reacting point absorber WEC designs. In Proceedings of the 10th European Wave and Tidal Conference (EWTEC), Aalborg, Denmark, 2-5 September 2013.

17. Chakrabarti, S.K. Hydrodynamics of Offshore Structures; WIT Press/Comp Mechanics: Wessex, UK, 1987.

18. Lopez Pavon, C.; Watai, R.A.; Ruggeri, F.; Simos, A.; Souto-Iglesias, A. Influence of wave induced second-order forces in semi-submersible FOWT mooring design. J. Offshore Mech. Arctic Engng. 2015, 137, 31602-31612. [CrossRef]

19. Lopez Pavon, C.; Souto-Iglesias, A. Hydrodynamic coefficients and pressure loads on heave plates for semi-submersible floating offshore wind turbines: A comparative analysis using large scale models. Renew. Energy 2015, 81, 864-881. [CrossRef]

(C) 2020 by the authors. Licensee MDPI, Basel, Switzerland. This article is an open access article distributed under the terms and conditions of the Creative Commons Attribution (CC BY) license (http:/ / creativecommons.org/licenses/by/4.0/). 